\title{
Transatlantica
}

Revue d'études américaines. American Studies Journal

$1 \mid 2012$

Le roman policier, littérature transatlantique / Maisons

Hantées

\section{Une histoire oubliée : la genèse française du terme « film noir » dans les années 1930 et ses implications transnationales}

Thomas Pillard

\section{(2) OpenEdition}

\section{Journals}

Édition électronique

URL : https://journals.openedition.org/transatlantica/5742

DOI : $10.4000 /$ transatlantica. 5742

ISSN : 1765-2766

Éditeur

Association française d'Etudes Américaines (AFEA)

Référence électronique

Thomas Pillard, « Une histoire oubliée : la genèse française du terme « film noir » dans les années 1930 et ses implications transnationales », Transatlantica [En ligne], 1 | 2012, mis en ligne le 14 décembre 2012, consulté le 01 février 2023. URL : http://journals.openedition.org/transatlantica/5742 ; DOI : https://doi.org/10.4000/transatlantica.5742

Ce document a été généré automatiquement le 1 février 2023

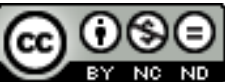

Creative Commons - Attribution - Pas d'Utilisation Commerciale - Pas de Modification 4.0 International - CC BY-NC-ND 4.0

https://creativecommons.org/licenses/by-nc-nd/4.0/ 


\title{
Une histoire oubliée : la genèse française du terme "film noir » dans les années 1930 et ses implications transnationales
}

\author{
Thomas Pillard
}

Selon l'historiographie du film noir américain, le terme générique "film noir » a été forgé en France en 1946 par des critiques comme Nino Frank et Jean-Pierre Chartier, pour caractériser des produits filmiques américains réalisés à partir du début des années 1940, comme Double Indemnity (Billy Wilder, 1944) ou The Postman Always Rings Twice (Tay Garnett, 1946). Depuis l'institution du genre consacré en 1955 par la parution $\mathrm{du}$ célèbre ouvrage de Raymond Borde et Étienne Chaumeton, Panorama du film noir américain (1941-1953), et l'installation de cette expression dans le vocabulaire de la critique anglo-américaine au début des années 1970, le terme « film noir » a connu une diffusion massive et une fortune discursive sans précédent, en restant solidement attaché au cinéma américain : il est de coutume de réserver cette dénomination aux seuls films hollywoodiens et de considérer le genre comme une spécificité américaine. Dans les différentes éditions de leur ouvrage Film Noir. An Encyclopedic Reference to the American Style (initialement paru en 1979, puis réédité en 1988 et en 1992), Alain Silver et Elizabeth Ward définissent par exemple le film noir comme « un ensemble de films indigènes au cinéma américain $»^{1}$ (Silver et Ward, 1992, 1).

Depuis le début des années 1990, certains chercheurs anglo-saxons ont pourtant entrepris de dissocier le genre de Hollywood, en s'intéressant aux ramifications européennes (et en particulier françaises et allemandes) du film noir américain. Charles O’Brien a ainsi montré que le terme «film noir » avait initialement été employé par la critique française, non en 1946 mais avant la Seconde Guerre mondiale, et pour parler d'un ensemble de films français, avant d'être réorienté après-guerre pour ne plus désigner que la production hollywoodienne (O'Brien, 1996). De son côté, Ginette Vincendeau a mis l'accent sur l'hybridité culturelle de ces films noirs français d'avant- 
guerre, en soulignant à quel point ils avaient bénéficié de l'apport des cinéastes et des techniciens émigrés allemands venus travailler dans les studios parisiens dans les années 1930, tout en démontrant qu'ils avaient constitué un antécédent fondamental pour la constitution du film noir américain (Vincendeau, 1992).

Ces travaux importants ont eu un impact certain sur la connaissance du film noir, mais la plupart des ouvrages consacrés au genre continuent paradoxalement de l'envisager comme une forme ontologiquement américaine. Ce point de vue a par exemple été réaffirmé par Alain Silver en 2010 dans l'introduction de la nouvelle édition refondue et augmentée de son ouvrage encyclopédique (plus sobrement intitulée Film Noir. The Encylopedia), où il explique que sa conception du film noir n'a pas changé depuis 1979:

Avec le western le film noir partage la distinction d'être une forme spécifiquement américaine, cela est aussi vrai maintenant qu'il y a trois décennies quand ce livre fut publié pour la première fois. [...] C'est un reflet de certaines préoccupations culturelles américaines sous une forme filmique. En bref, c'est un exemple unique d'un style de film entièrement américain ${ }^{2}$ (Silver, Ward, Ursini et Porfirio, 2010, 15).

Les études portant sur les origines européennes du film noir restent par ailleurs méconnues, voire inconnues, au sein de la recherche française, ce qui explique que ce phénomène générique y soit encore largement perçu comme purement américain. Dans l'ouvrage qu'elle a consacré à Jean-Pierre Melville, Denitza Bantcheva écrit par exemple :

Personne n'ignore que le film noir est avant tout américain : indiscutablement, les grands classiques du genre relèvent d'une culture nationale spécifique où il a pu prendre une place non négligeable avant d'exercer une influence sensible sur les cinématographies européennes et autres (Bantcheva, 2007, 119).

Il n'est évidemment pas contestable que les films noirs américains soient fortement enracinés dans la culture américaine ainsi que dans l'histoire des États-Unis, et qu'ils aient pu avoir un impact important sur d'autres cinématographies. Néanmoins ce que " personne n'ignore » sur les frontières géographiques et culturelles du genre dissimule en réalité une histoire oubliée, considérablement plus complexe, qui n'a pour le moment été qu'esquissée, et dont il reste à faire émerger la caractéristique la plus notable, à savoir sa dimension internationale : non seulement le terme «film noir » a été utilisé à propos du cinéma français avant que le genre ne devienne le parangon du cinéma américain classique, mais il a initialement été employé par la critique hexagonale pour désigner des films se rattachant à une forme filmique cosmopolite.

Dans cette perspective, cet article se propose de revenir sur la genèse française de cette célèbre appellation générique dans les années 1930, avant d'interroger sa signification pour la critique de l'époque et de dégager ensuite ses implications transnationales.

\section{La genèse française du terme «film noir » dans les années 1930 et sa « réorientation culturelle » après la Libération}

7 Dans un article intitulé «Film Noir in France : Before the Liberation », paru en 1996 dans un numéro de la revue Iris consacré aux " Précurseurs européens du film noir », l'historien Charles O'Brien a été le premier à montrer que le terme « film noir » a été à l'origine employé par la critique française avant la Seconde Guerre mondiale à propos du cinéma français. 
O'Brien se fonde sur les comptes rendus critiques parus dans la presse entre le mois de janvier 1938 - date à partir de laquelle l'étiquette générique est utilisée de façon récurrente et précise pour désigner un "genre spécifique " (O’Brien, 1996, 7) - et le mois de septembre 1939 - moment où l'entrée en guerre de la France amène la presse à cesser de publier des critiques de films. Il montre que l'appellation « film noir » émerge durant cette période pour désigner un corpus d'au moins treize films français réalisés entre 1935 et 1939, parmi lesquels figurent plusieurs films réalisés par Marcel Carné et/ ou reposant sur la performance de la star Jean Gabin :

Crime et châtiment (Pierre Chenal, 1935

Jenny (Marcel Carné, 1936)

Les Bas-fonds (Jean Renoir, 1936)

Pépéle Moko (Julien Duvivier, 1937, avec Jean Gabin)

Le Puritain (Jeff Musso, 1938)

L'Étrange Monsieur Victor (Jean Grémillon, 1938)

Quai des brumes (Marcel Carné, 1938, avec Jean Gabin)

Hôtel du nord (Marcel Carné, 1938)

La Bête humaine (Jean Renoir, 1938, avec Jean Gabin)

La Tradition de minuit (Roger Richebé, 1939)

Le Dernier tournant (Pierre Chenal, 1939) ${ }^{3}$

Quartier sans soleil (Dimitri Kirsanoff, réalisé en 1939, sorti en 1945)

Le jour se lève (Marcel Carné, 1939, avec Jean Gabin)

Ce corpus, qui rassemble à la fois des œuvres célèbres et des titres méconnus, correspond plus ou moins à ce que l'on appelle aujourd'hui le "réalisme poétique " $^{4}$. Selon plusieurs sources (Andrew, 1995, 11 ; Phillips, 2004, 118), ce terme ambigu aurait été forgé par Jean Paulhan à propos du roman La Rue sans nom (1930) de Marcel Aymé5, avant d'être repris par Michel Gorel en 1934 pour qualifier l'adaptation de ce roman par le cinéaste Pierre Chenal : «Ce film, à mon sens, inaugure dans le cinéma français un genre entièrement nouveau : le réalisme poétique et "actuel” " (Gorel, 1934, 114). S'il a tardé à s'imposer dans le vocabulaire courant, ce vocable a cependant connu une grande fortune discursive depuis les années 1970, qui a longtemps contribué à masquer les enjeux idéologiques des films, surtout en France. La popularité de l'expression "réalisme poétique », le poids de l'interprétation artistique des films en France et les réticences de la critique hexagonale à penser le cinéma français en termes génériques (Moine, 2007) ont rendu difficile l'identification du « réalisme poétique » comme genre et comme "équivalent générique français » du film noir américain, malgré le fait que plusieurs films noirs américains des années 1940 et 1950 sont des remakes de films français réalisés dans les années $1930^{6}$.

11 Dans les années 1938-1939, l'utilisation de l'expression «film noir » est réservée à des productions françaises, et porte des connotations péjoratives. L'étiquette est en effet presque toujours utilisée par les critiques pour dénoncer le ton pessimiste, le caractère immoral et l'atmosphère démoralisatrice de ces films réalisés à la veille de la Seconde Guerre mondiale, comme le montre cet extrait de l'éditorial du quotidien Le Petit Journal, réagissant à l'attribution du « Grand Prix national du cinéma français » au film Quai des brumes de Marcel Carné :

Il est désolant de voir le plus officiel des prix cinématographiques français, le prix du ministère, attribué à un film, plein de qualités artistiques, certes, mais d'un genre très spécial. Un film noir, un film immoral et démoralisant, dont les effets, 
auprès du public n'ont pu qu'être néfastes (Anonyme, 8 juillet 1939, cité dans

O’Brien, 1996, 10). idéologique résolument distinct de la culture cinématographique cinéphile de l'aprèsguerre, qui procédera à l'invention du «film noir hollywoodien » afin d'en célébrer "l'atmosphère insolite et cruelle, teintée d'un érotisme assez particulier " (Borde et Chaumeton, 2004, 11). Issues d'un contexte très différent, les références au « film noir » à la fin des années 1930 s'inscrivent dans le cadre d'un "journalisme culturel politisé » (O’Brien, 1996, 10). Elles apparaissent principalement non dans des magazines spécialisés sur le cinéma mais dans des journaux accordant beaucoup moins d'attention aux films qu'à la politique et à l'économie - la situation s'inversera après-guerre, puisque le terme "film noir» commencera à être employé à propos du cinéma américain dans des revues comme L'Écran français et La Revue du cinéma. Enfin elles proviennent surtout de la presse d'extrême droite : on trouve en particulier différentes occurrences du terme dans les articles publiés dans le quotidien L'Action française par Lucien Rebatet (sous le pseudonyme de François Vinneuil), célèbre critique et figure intellectuelle importante de l'époque ${ }^{7}$, fasciste autoproclamé, et pourfendeur régulier du film noir français.

Le fait que le terme « film noir » soit à l'époque réservé aux films français, et qu'il soit porteur d'une connotation péjorative, rend d'autant plus surprenant son remploi après la Libération, toujours par la critique française, mais cette fois-ci pour désigner, dans une intention ouvertement moins polémique, voire de célébration, des films américains. Durant l'immédiat après-guerre, le terme «film noir » est dans un premier temps employé à propos du cinéma américain en référence à la tradition française d'avant-guerre-un des premiers articles consacrés au film noir américain, écrit par Jean-Pierre Chartier, s'intitule " Les Américains aussi font des films "noirs" " (Chartier, 1946, 67-70). Mais il cesse progressivement de renvoyer au cinéma hexagonal, pour ne plus venir désigner (et généralement mettre en valeur), à partir de la deuxième moitié des années 1950, que la seule production hollywoodienne.

Un examen approfondi des critiques de films publiées à l'époque révèle que le terme « film noir » a continué pendant plusieurs années à être employé à propos de certaines œuvres hexagonales, parallèlement à son nouvel usage américain, par des critiques de tous bords politiques : des films comme Quai des orfêvres (Henri-Georges Clouzot, 1947) et Manèges (Yves Allégret, 1950) ont parfois été qualifiés de « films noirs » et reliés à la tradition française d'avant-guerre ${ }^{8}$. Il n'en demeure pas moins que se met en place à la même époque, dans les discours tenus sur le film noir américain, une stratégie argumentative visant à refuser au cinéma français un terme désormais prioritairement associé à Hollywood, qui va peu à peu parvenir à ses fins, et dont les effets se font à l'évidence encore sentir aujourd'hui. Ce clivage est « scellé » en 1955 par la parution du Panorama du film noir américain de Borde et Chaumeton, un ouvrage s'inscrivant clairement dans un héritage intellectuel proche du surréalisme (voir Naremore, 1998, 18-22), dont les thèses connaîtront - malgré leur caractère souvent approximatif - un impact considérable sur l'ensemble des discours postérieurs tenus sur le film noir 9 . Les auteurs y consolident définitivement cette application restrictive de l'étiquette générique aux seuls films hollywoodiens, en définissant le film noir comme une "série ", c'est-à-dire, dans leur acception du terme, comme "un ensemble de films nationaux ayant entre eux quelques traits communs (style, atmosphère, sujet...) assez forts pour les marquer sans équivoque » (Borde \& Chaumeton, 2004, 12). Pour Borde et 
Chaumeton, qui concèdent au détour d'une phrase que Duvivier, Renoir et Carné " avaient créé un certain réalisme noir " (Ibid., 34) dans le cinéma français d'avantguerre, il n'est en effet pas pertinent de relier le film noir américain des années 1940 et 1950 aux films français sombres et mélodramatiques des années 1930, au motif que ces derniers exprimeraient une tradition réaliste :

Pépé le Moko, Quai des brumes, La Bête humaine n'annonçaient-ils pas le film noir américain? Nous ne le pensons pas. D'abord l'onirisme et l'insolite font complètement défaut dans ces films des années 1936-1938. Dès le début, l'action est située dans un milieu social bien défini (Ibid., 34-35).

L'examen de cette surprenante réorientation culturelle dépasse le cadre et les ambitions de cet article. Nous soulignerons donc simplement ici avec Ginette Vincendeau (1992, 49) que l'argument principal des deux auteurs semble rétrospectivement très contestable, voire fallacieux : il présente comme un élément de preuve irréfutable que le film noir américain n'entretient aucun rapport avec le «réalisme noir» français le fait que ce dernier, justement, est plus réaliste et ne possède pas l'aspect onirique qui marque tant de films noirs américains... alors que le " réalisme » est simplement une spécificité stylistique propre au cinéma français dans son ensemble ${ }^{10}$, qui n'empêche pas les films d'avant-guerre de partager de nombreuses similitudes stylistiques, iconographiques, narratives et thématiques avec les nouveaux films criminels hollywoodiens des années 1940 et 1950.

La définition du film noir proposée par Borde et Chaumeton traduit en outre un glissement idéologique, comme le suggère O'Brien en exprimant, à l'aide des travaux de Pascal Ory $(1989,126-152)$, l'idée que cette réorientation culturelle apparaît comme une conséquence du développement artistique et intellectuel de la France d'après-guerre. Immédiatement après la Libération, il serait devenu idéologiquement nécessaire, pour les artistes et les intellectuels de la génération 1944-1955, de reconstruire une culture nationale spécifique en cherchant à réhabiliter les mouvements avant-gardistes des années 1920 et de la première moitié des années 1930 (au premier rang desquels le surréalisme), et en établissant une distance avec la culture dominante de la fin des années 1930. O'Brien précise que l'esprit des films noirs français d'avant-guerre était d'autant plus inconciliable avec ce positionnement culturel qu'ils semblaient dorénavant «marqués de façon indélébile, incarnant pour toujours une mentalité maintenant identifiée comme étant la principale cause de la défaite nationale » (17). Il y a de ce point de vue une forme de logique et de continuité, entre l'utilisation du terme « film noir » dans les années 1930 et le refus de l'employer que l'on constate aprèsguerre, à l'heure de la reconstruction nationale.

Ce phénomène a eu pour effet de créer une rupture a priori entre les cinématographies françaises et américaines, d'associer de façon pérenne l'idée de "film noir » avec l'imaginaire américain, et de cristalliser pour la postérité une topographie du genre, qu'il est temps aujourd'hui de réévaluer.

\section{Qu'est-ce qu'un film noir français dans les années 1938-1939?}

18 Si le travail de Charles O'Brien constitue un apport indéniable et fondamental, une étude complémentaire des discours de réception portant sur les films qualifiés de «films noirs" par la presse française à la fin des années 1930 s'avère toutefois 
nécessaire pour mettre à jour la signification de la construction discursive « film noir " à cette époque. Dans cette perspective, nous proposons de mener une étude pragmatique ${ }^{11}$ semblable à celle que Rick Altman préconise dans son ouvrage Film/Genre (2006). Altman explique qu'une telle approche refuse de considérer les genres comme des ensembles fixes déterminés en amont et comme des catégories préexistantes à leur réception: il s'agit au contraire de les envisager comme des "lieux de lutte et de coopération " (Ibid., 211) entre différents usagers d'une même étiquette générique, dont le sens est indissociable de la variété des lectures qu'ils peuvent susciter au sein d'une communauté dans un contexte historique donné.

19 L'étude des discours tenus sur le film noir français dans les années 1938-1939 montre que ces derniers se déploient dans quatre directions principales : l'idéologie; le style visuel; les caractéristiques narratives; l'identité culturelle. Dans la mesure où l'idéologie des films à déjà été commentée en détail par O'Brien, nous laisserons cet aspect de côté pour examiner plus avant les trois autres enjeux discursifs qui donnent à cette période son sens au " film noir", en tentant dans chacun des cas d'établir des comparaisons avec les discours tenus sur le film noir américain à partir de 1946.

\section{Un style visuel « noir »}

Considéré depuis les années 1970 comme un des traits les plus fortement identifiants du film noir américain ${ }^{12}$, le style visuel était déjà avant-guerre un des éléments les plus remarqués dans les films noirs français, comme l'indique l'emploi courant de l'étiquette générique alternative "film d'atmosphère» pour désigner les mêmes films. Les critiques soulignent presque toujours l'intense sophistication du style et associent la notion d'ambiance visuelle à l'identité du genre : dans Les Nouvelles littéraires, Alexandre Arnoux écrit par exemple, à propos de Carné, que " peu de metteurs en scène ont, à ce degré, le don de faire l'ambiance " (Arnoux, 17 juin 1939). Plus encore, peut-être en raison des connotations très anciennes associées à la couleur noir dans la culture occidentale $^{13}$, l'atmosphère sombre et ténébreuse des films semble avoir joué un rôle important dans l'élaboration et la consolidation de l'étiquette générique. Les critiques sont en effet nombreux à souligner la noirceur inhabituelle ou excessive des films (figure 1), à l'instar de Rebatet, qui écrit dès 1936 à propos de Jenny que le film est « gâté par un parti-pris assez bizarre d'obscurité » (Vinneuil, 16 octobre 1936). Plus largement, il est fréquemment fait allusion au "sombre lyrisme $»^{14}$ des films, à leur 《sombre poésie $~^{15}$ ou encore à «leur technique sobre et un peu noire » (Arnoux, 17 juin 1939). 


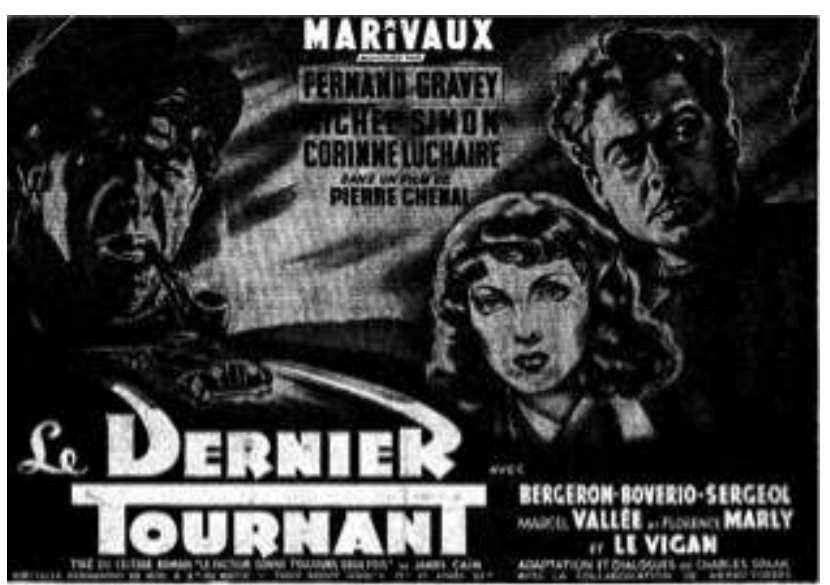

Le Dernier tournant (Pierre Chenal, 1939). Ce document publicitaire d'époque traduit bien à la fois la noirceur excessive du film de Chenal et l'importance de l'atmosphère visuelle dans le choix du terme " film noir ».

Source : L'Intransigeant, 18 mai 1939. Bibliothèque nationale de France.

Ce style visuel «noir » est le plus souvent décrit comme « réaliste »: Serge Veber écrit par exemple, à propos de $L a$ Bête humaine, que "ce réalisme par l'image est extrêmement pénible " (Veber, 28 décembre 1938, 6). Les critiques notent toutefois régulièrement en parallèle l'artificialité, la "poésie » et le «lyrisme» de certaines séquences, en soulignant l'apport esthétique des chefs-opérateurs allemands et en faisant le lien avec la sophistication visuelle du cinéma de Weimar. Cela les conduit parfois à souligner le caractère paradoxal de l'esthétique du genre, comme dans cette remarque d'un critique de Pour vous sur Le Puritain : «Le tout est haché, morcelé, avec des artifices sur un fond naturel. Il en résulte des effets boiteux, hétéroclites » (L.W., 19 janvier 1938, 5). Le terme "film noir» renvoie donc à l'époque à un style évocateur mais difficile à définir, qui oscille entre une tendance globale au " réalisme » et un goût plus ponctuel pour l'abstraction lyrique hérité du cinéma allemand.

On retrouvera de manière frappante des descriptions similaires, et dans certains cas identiques, à partir de 1946, dans les articles français consacrés au film noir américain : Jean-Pierre Chartier décrit les spécificités de Murder, my Sweet (Edward Dmytryk, 1944) en soulignant «l'obscurité du film»(Chartier, 1946, 69); Nino Frank évoque l'impression de réalité que dégagent le style des films américains en écrivant que ce style accentue le "côté vécu " (Frank, 1946, 14); dans un article sur "Le style germanique à Hollywood ", Amable Jameson évoque le style visuel hybride des films noirs américains réalisés par Lang et Siodmak, deux cinéastes qui a l'instar de Wilder avaient travaillé dans les studios parisiens avant d'émigrer aux États-Unis ${ }^{16}$ : il écrit que «Fritz Lang montre les aspects tragiques de la vie américaine dans cette lumière dure et tranchante qu'il a apportée de Berlin », et parle de "couleur spéciale " (Jameson, 1947, 65) à propos de The Killers (Robert Siodmak, 1946), utilisant à cette occasion une expression fréquemment employée dans les années 1930 à propos des films noirs français ${ }^{17}$.

\section{Conventions narratives : une forme spécifique de récit criminel}

23 L'appellation "film noir» sert également aux critiques à rassembler des films proposant des intrigues similaires et reposant sur ce qui est parfois perçu comme une «formule industrialisée $»^{18}$. Trois traits principaux ressortent en particulier des 
discours : l'importance du crime et de la mort; les différences narratives entre le film noir et le whodunit; et enfin la nature mélodramatique, pessimiste et tragique de ces drames criminels et passionnels.

L'étiquette « film noir » renvoie d'abord à l'époque à des intrigues où le crime possède une place et une fonction déterminante, comme l'indique ce commentaire de Rebatet à propos du Puritain: "M. Jeff Musso a choisi un sujet classique : le film noir, plongeant dans la débauche et le crime $»^{19}$ (Vinneuil, 21 janvier 1938). Les commentateurs mettent tout d'abord l'accent sur le nombre anormalement élevé de meurtres qu'il peut y avoir à l'intérieur d'un même film : à propos de La Bête humaine, Serge Veber note que " trois morts, c'est beaucoup pour un seul film » (Veber, 28 décembre 1938). Un autre critique, plus virulent, reproche au film de Renoir de ne pas donner à voir autre chose qu'une série d'assassinats et de présenter le meurtre comme un acte «naturel»:

Il ne s'agit d'un bout à l'autre de ce film que de meurtres et d'assassinats. Tous les personnages : le sous-chef de gare assassin et voleur, la femme p... et meurtrière, le riche bourgeois satyre et assassiné et, brochant sur le tout, le sympathique mécanicien criminel. 嚂...㜯 Le meurtre dans ce film est monnaie courante. «Tuele !» « Pourquoi ne le tues-tu pas? " «Je l'ai tué » sont les principales finesses du dialogue. 熙...器 Aucun film de gangsters américains dont on a justement déploré la nocivité ne peut avoir une plus pernicieuse influence que ce film malsain où le crime est non pas exalté, mais présenté comme la chose au monde la plus naturelle (de Montgon, 28 janvier 1939).

Comme le montre bien cet exemple, les critiques soulignent aussi l'importance du crime dans l'économie narrative globale des films. Un film noir comme Le jour se lève (qui débute par le meurtre de Jules Berry par Jean Gabin, et dont la construction en flash-back anticipe l'utilisation que feront de ce procédé les films noirs américains) est un film « où le mal et la mort violente exercent de tristes ravages $»^{20}$ (N.F., 14 juin 1939, 5) et où les critiques ont immédiatement conscience de l'importance et du poids particulier du meurtre initial, comme le laisse transparaître cette remarque de James de Coquet, racontant l'intrigue du film à ses lecteurs: «Ce meurtre, auquel nous venons d'assister, c'est le dénouement du film » (de Coquet, 14 juin 1939).

On voit ici que l'étiquette "film noir" ne constitue pas pour les critiques un simple synonyme de l'expression « film criminel» : elle ne sert pas à rassembler des films où un crime a lieu à un moment du récit (ce qui pourrait correspondre à une définition large et extensive du genre criminel), mais plutôt des films où l'acte de tuer et le fait de mourir sont des événements narratifs à la fois probables, "sérieux " et décisifs, sur lesquelles reposent véritablement les intrigues des films ainsi que leur ampleur dramatique. Or si pour les critiques d'avant-guerre, le crime apparaît comme l'un des éléments les plus fermement associé au "film noir ", ce sera toujours le cas aprèsguerre. Jean-Pierre Chartier débute par ainsi son article par une remarque sur ce qu'il considère être l'argument narratif principal des films noirs américains-«Elle l'embrasse pour qu'il tue »-et stigmatise les intrigues meurtrières et «l'obsédante fatalité du crime» (Chartier, 1946, 68) qui conditionne les personnages de Double Indemnity et Murder, my Sweet de manière tout à fait comparable aux discours tenus dans les années 1930 sur le film noir français. Dès les premières pages de leur ouvrage, Borde et Chaumeton écrivent de leur côté que

c'est la présence du crime qui donne au film noir sa marque la plus constante. [...] Peu de séries dans l'histoire du cinéma ont accumulé en sept ou huit ans autant de brutalités crapuleuses et d'assassinats. [...] À tous les sens du mot, le film noir est un film de mort (Borde et Chaumeton, 2004, 15-16). 
D'autre part, bien que cet aspect n'apparaisse pas de manière extrêmement fréquente dans les comptes rendus de l'époque, les « films noirs » de la fin des années 1930 sont parfois rattachés à la plus vaste catégorie du « film policier ", tout en étant considérés comme faisant partie d'un ensemble plus spécifique, distinct de la tradition populaire moins subversive, plus « grand public » et quantitativement plus importante du film à énigme ou whodunit. La forme générique à énigme, qui " prend son essor au début du parlant» (Berthé-Gaffiero, 2007, 204) et dont Le Mystère de la chambre jaune (Marcel L'Herbier, 1930) constitue un exemple emblématique, rassemble des films «qui comportent un crime dont l'auteur ne sera désigné qu'à la fin » (Ibid.). Ce trait distinctif permet à Emmanuel Boudot-Lamotte d'insister, à l'instar d'autres critiques de l'époque, sur la différence narrative qu'un film noir comme Le Puritain entretient avec le whodunit, en notant que le film de Musso

s'apparente, en effet, au genre du film policier, puisqu'il commence par un crime, se poursuit par une enquête et s'achève sur l'arrestation du coupable. Et cependant, c'est le contraire d'un film policier, puisqu'il n'y a pas d'énigme à résoudre: le spectateur connaît le coupable depuis le début (Boudot-Lamotte, 7 décembre 1940).

Cette différence entre les deux genres semble si évidente qu'on peut imaginer que les critiques n'aient pas ressenti le besoin d'insister sur cet aspect dans leurs commentaires. Elle n'en est pas moins importante, pour au moins deux raisons : d'une part, parce que les spécialistes du roman noir américain ont montré que celui-ci s'était précisément constitué en opposition au roman à énigme "à l'anglaise", sur le plan linguistique comme au niveau narratif (Tadié, 2007, 27-64); d'autre part, parce que cette argumentation réapparaîtra de manière absolument identique sous la plume des commentateurs français à partir de 1946 pour exprimer ce qui ferait la spécificité de la "Série Noire » littéraire et du film noir en tant que « nouveaux genres » américains, en rupture avec la fiction policière traditionnelle ${ }^{21}$. Si un critique d'après-guerre rappelle, en prenant appui sur un film de Chenal, que cette distinction entre roman/film noir et forme à énigme n'est pas nouvelle ${ }^{22}$, la majorité des commentateurs, dans un mouvement d'occultation de masse saisissant, vont pourtant l'interpréter, à l'instar de Marcel Duhamel et de Nino Frank, à la fois comme une nouveauté radicale et comme un trait spécifiquement américain, comme si le film noir français d'avant-guerre n'avait jamais existé. Les critiques emploient enfin la dénomination " film noir " pour rassembler dans une même catégorie des drames criminels et passionnels reposant sur une association entre le crime et le désir, et qui associent le registre émotionnel du mélodrame à un «mécanisme tragique » condamnant les protagonistes masculins à un destin fatal ${ }^{23}$.

On constate ainsi une forte association dans les discours entre les films noirs et le mélodrame, qui constitue de longue date un genre bien implanté dans le cinéma français ${ }^{24}$. Le terme de "mélodrame» est employé par les critiques, de façon généralement péjorative, pour préciser le registre émotionnel des films, mettre en évidence l'importance de la passion amoureuse dans les récits, et souligner le poids de la fatalité et la passivité des personnages. Émile Vuillermoz consacre par exemple de longues lignes au registre mélodramatique d'Hôtel du nord, qu'il qualifie à la fois de «nouveau film poussé au noir», de «drame de l'amour et de la mort» et de «mélodrame de carrefour»:

Nous sommes là en présence d'une race d'hommes et de femmes plus résignée que les autres aux caprices de la fatalité. Il faut remonter à la tragédie grecque et aux personnages d'Eschyle pour retrouver pareille obéissance passive aux arrêts du 
destin. Et c'est ce qui donne à ces mélodrames de carrefour une dangereuse noblesse (Vuillermoz, 14 janvier 1939).

31 Le lien avec le mélodrame est consolidé par l'insistance avec laquelle les critiques stigmatisent la passivité des protagonistes. Cet aspect des films est peut-être celui qui déclenche les commentaires les plus violents. Rebatet compare ainsi fréquemment les personnages de victimes interprétés par Gabin à des insectes ou à des "larves ». En 1939, il commente l'incapacité à agir des personnages du Dernier Tournant en mobilisant ce type de vocabulaire et en rattachant ce trait narratif emblématique du mélodrame à l'identité générique du film noir :

Plusieurs jeunes metteurs en scène français, pour réagir contre la frivolité $d u$

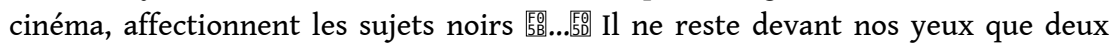
misérables larves, inconscientes, livrées à leurs instincts qui n'ont même pas une sorte de puissance sauvage, pataugeant stupidement dans le sang. Ils n'éveillent en nous qu'un seul sentiment: le désir de les voir le plus vite possible éliminés par n'importe quel moyen de la société (Vinneuil, 19 juin 1939).

On voit ici que dans l'esprit des critiques le mot « noir » ne renvoie pas seulement à un style, mais aussi à un modèle dramaturgique spécifique, qui impose à des personnages passifs et impuissants de sombrer dans le crime. Cet aspect des films irrite en particulier la presse d'extrême droite, pour qui une telle peinture de l'impuissance (masculine) est inconciliable avec l'idée qu'ils se font de la France (un pays « sain » et vigoureux)... et de la masculinité : dans sa critique d'Hôtel du nord, Rebatet compare les héros des films de Marcel Carné à des " débris descendants le canal Saint-Martin » et constate «qu'on ne peut rien imaginer de moins viril » (Vinneuil, 30 décembre 1938). On trouve toutefois certains articles allant dans le même sens dans la presse de gauche, comme le montre par exemple cette critique virulente du Dernier tournant parue dans Marianne durant l'été 1939 :

Voici encore un film noir, un film de cette série sinistre qui part de Les Bas-fonds et de Crime et Châtiment et se poursuit par Pépé le Moko et Quai des brumes, La Bête Humaine et Hôtel du Nord. [...] Nous commençons à être las de cette atmosphère spéciale, de ces espoirs voués à l'échec, de ces personnages que leur implacabledestin conduit vers la déchéance et la mort. Il est temps que l'écran français s'éclaire (Mezzanine, 31 mai 1939).

Les films noirs français trouvent ici une parenté solide avec les films noirs américains, dont les implications mélodramatiques ont été notées dès 1946 par plusieurs critiques (voir par exemple Fayard, 19 avril 1947), et qui ont depuis fait l'objet d'analyses spécifiques et détaillées: Frank Krutnik a montré que le film noir américain s'apparentait à une forme de mélodrame masculin, dont les personnages masochistes témoignent d'une «fascination persistante pour le spectacle de l'homme passif ou émasculé » (Krutnik, 2004, 128), et d'une profonde crise de la masculinité dans la société américaine des années 1940.

Le terme de "tragédie » et l'adjectif « tragique » sont parfois employés par les critiques à la fin des années 1930, précisément pour ajouter aux implications mélodramatiques l'idée d'un fort pessimisme et d'une radicalité tragique - contrairement au mélodrame, où la fatalité peut se concilier avec la logique du happy-end, «la stricte syntaxe de la

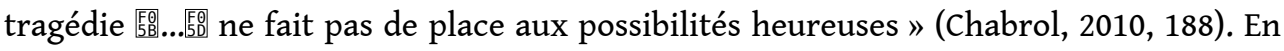
raison du capital culturel élevé attaché à la notion de tragédie, le terme est toutefois généralement convoqué pour être aussitôt refusé aux films, comme lorsque Rebatet écrit que les choix de cinéastes comme Carné et Chenal «se portent toujours sur les plus vulgaires faits divers, et ils restent incapables de les élever jusqu'au rang de la 
tragédie » (Vinneuil, 19 mai 1939). Ce refus manifeste la volonté de commentateurs tels que Rebatet de maintenir les films noirs français dans la sphère d'une culture populaire illégitime et méprisable, avec laquelle le terme de « mélodrame » reste solidement lié. Il est de ce point de vue notable de constater que les discours tenus sur le film noir américain à partir de 1946 s'efforceront eux aussi de décrire un type de fiction criminelle populaire et mélodramatique, mais en cessant pour la plupart de considérer leur noirceur de façon négative ${ }^{25}$ (le texte de Chartier est à cet égard une exception), et en hésitant cette fois-ci beaucoup moins à la qualifier de "tragique " et à l'élever au rang d'« art »: dans une critique consacrée à The Woman in the Window (1946) Jacques Bourgeois écrit par exemple que «Fritz Lang n'a jamais traité qu'un seul sujet, le sujet tragique fondamental: l'homme vaincu par son destin après une lutte qui constitue la péripétie dramatique de l'œuvre [...] et il s'agit bien d'une tragédie où Fritz Lang nous entraîne tout entier » (Bourgeois, 1946, 70-72).

\section{Une forme générique transnationale}

Pour les critiques des années 1938-1939, les films réunis sous la dénomination « film noir » forment un ensemble particulier également parce qu'ils possèdent une identité culturelle spécifique. Deux aspects ressortent particulièrement: la dimension intermédiatique des films et, surtout, leur dimension internationale et cosmopolite. Les critiques soulignent en effet régulièrement la forte hybridité culturelle des films, c'està-dire le fait qu'ils se constituent à travers un mélange d'éléments perçus comme nationaux et d'éléments perçus comme étrangers, en provenance de l'Allemagne et des États-Unis. D'un côté, ils relèvent le fort ancrage de la plupart des films dans la tradition du Roman Populiste, importante école littéraire française des années 1920 incarnée par des auteurs comme Pierre Mac Orlan et Eugène Dabit, dont le «petit peuple » est le thème central, et dont les films noirs de la fin des années 1930 sont défavorablement perçus comme étant les héritiers : à propos du Jour se lève, Jean Fayard écrit par exemple dans Candide que "sous couleur de populisme, on veut nous faire avaler la pire littérature » (Fayard, 28 juin 1939) ${ }^{26}$. D’un autre côté, les mêmes films sont souvent mis en relation avec des œuvres étrangères.

\section{L'impact du cinéma allemand}

36 L'interprétation dominante à la fin des années 1930 consiste à considérer ces films noirs français comme des héritiers de certains films allemands, et en particulier de deux types de drames « réalistes » et mélodramatiques des années 1920 et du début des années 1930: le Strassenfilme ("film de rue », en français) et le Kammerspielfilm (expression que l'on pourrait traduire par « film de chambre ») ${ }^{27}$. Faisant référence à un film de Pabst qui constitue un exemple emblématique de la première de ces deux catégories, Rebatet écrit à propos de Pépé le Moko que «Duvivier n'a pu échapper complètement aux souvenirs de "la rue sans joie" des Allemands, qui poursuit les cinéastes sous tous les climats dès qu'ils abordent les lieux de la prostitution et du crime $\aleph^{28}$ (Vinneuil, 5 février 1937). Jean Fayard qualifie quant-à-lui Le jour se lève de « caricature de l'ancien film allemand » (Fayard, 28 juin 1939).

L'identification de cette filiation, facilitée par la présence de nombreux techniciens allemands dans les équipes techniques des films, est motivée par trois facteurs. Le 
premier est lié à l'iconographie et en particulier aux motifs visuels liés au décor : la topographie urbaine de la "zone », du faubourg ouvrier ou des "bas-fonds », filmée de préférence la nuit, avec ses rues mal éclairées, ses pavés mouillés, ses lampadaires brillant dans la nuit et ses chambres miteuses dans lesquelles les personnages se replient souvent sur eux-mêmes. Un critique écrit par exemple à propos du Puritain que, "si le sujet est d'origine irlandaise et traité en langue française, le film se développe parmi des décors qui s'apparentent au réalisme de l'ancien cinéma berlinois » (L.W., 19 janvier 1938). La seconde explication est d'ordre narrative : les critiques considèrent souvent que les films noirs français proposent le même type d'intrigues que certains films allemands plus anciens. Émile Vuillermoz emploie ainsi précisément l'adjectif «noir» (sans connotation péjorative, ce qui est assez rare pour l'époque) pour établir un rapprochement entre le film noir français et le cinéma allemand :

Le sujet est noir. C'est, actuellement, la couleur à la mode dans nos studios. Nos cinéastes nous donnent, en ce moment, des films qui font penser à ceux qu'on tournait à Berlin il y a quelques années. L'authentique chef d'œuvre qui s'appelle Quai des brumes traduit la même hantise morbide que l'admirable Rue sans joie (Vuillermoz, 14 janvier 1939).

Enfin, les comparaisons France/Allemagne sont également liées à l'atmosphère visuelle des films français, qui oscille comme les drames allemands entre un "réalisme » global sombre et austère, et une tendance à créer de façon plus ponctuelle des moments d'abstraction lyrique reposant sur des éclairages plus artificiels et plus sophistiqués. Rebatet fait souvent ce rapprochement pour dénoncer ce qu'il appelle le "pseudoréalisme » du genre, dans des commentaires qui laissent ouvertement transparaître son antisémitisme, comme ici à propos d'Hôtel du Nord : « nous avons vu ce pseudo-réalisme

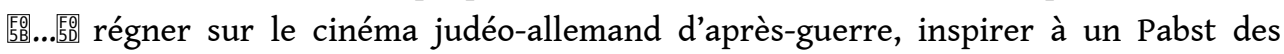
tableaux parfois puissants, mais presque toujours au service d'une thèse stupide " (Vinneuil, 30 décembre 1938). Durant l'Occupation, à l'occasion de la reprise du Jour se lève dans une salle parisienne, il écrira de manière plus violente encore à propos de Carné :

J'ai maintes fois écrit, avant la guerre, que ses films procédaient d'une esthétique fort haïssable et qui datait déjà beaucoup lorsqu'elle connut chez nous un regain de faveur grâce aux transfuges juifs de l'Europe centrale qui avaient mis, durant ces dernières années, la haute main sur notre cinéma (Vinneuil, 5 janvier 1941).

Les critiques d'extrême droite condamnent le style « noir » parce qu'il symbolise à leurs yeux la dimension cosmopolite du genre. Révélateurs du climat idéologique nauséabond de l'époque, de tels propos n'en indiquent pas moins que de nombreux commentateurs voyaient dans le film noir un ensemble culturellement hybride. Or le film noir américain sera décrit de façon très similaire à partir de 1946, en particulier par les critiques communistes cherchant à soulignant la capacité des cinéastes et des chefs-opérateurs émigrés européens à insuffler une dimension artistique à la production de masse impersonnelle de Hollywood. C'est notamment le cas dans ce qui est à notre connaissance le premier article à employer après-guerre le terme de « film noir " à propos du cinéma américain : un compte-rendu de Jacques Borel paru dans L'Écran français en juin 1946 et intitulé "Hollywood n'a pas standardisé Fritz Lang " 29. Dans une critique de Scarlet Street, qu'il a pu voir à Londres avant que le film ne sorte sur les écrans parisiens, Borel note que le film fait partie d'« une fournée de films 
noirs » qui arrive sur les écrans, avant d'affirmer que le cinéaste allemand a su « impos臨er

\section{Une proximité avec l'Amérique}

Les films noirs français sont parfois mis en relation avec la littérature américaine et le cinéma américain. C'est évidemment à propos du Dernier Tournant, adaptation d'un roman de James Cain, que cette relation France/Amérique apparaît le plus clairement. Les critiques, qui mettent souvent l'accent sur la fidélité de l'œuvre de Chenal par rapport au roman, expriment des sentiments ambivalents à l'égard de cette source littéraire étrangère. Rebatet fait preuve d'un antiaméricanisme violent et teinté

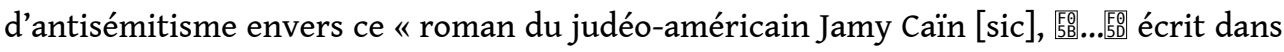
l'effroyable jargon du réalisme yankee » (Vinneuil, 19 juin 1939). Mais les réactions sont loin d'être unanimement négatives, même dans la seule presse d'extrême droite, comme en témoigne par exemple la critique de Georges Champeaux parue dans la revue ouvertement fasciste et antisémite Gringoire, qui ne s'attaque qu'au travail du metteur en scène (ne le jugeant pas à la hauteur de l'œuvre de Cain) et non au roman initial ou à l'adaptation :

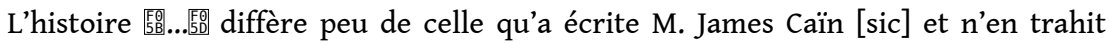
jamais l'esprit. L'adaptation de MM. Charles Spaak et Henry Torrès est à la fois fidèle et ingénieuse. Malheureusement le découpage de M. Chenal est, comme

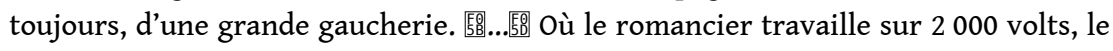
metteur en scène travaille sur 110. Il fallait se sentir capable de faire aussi brutal, aussi véhément que Scarface, ou ne pas s'en mêler (Champeaux, 25 mai 1939).

Il ne s'agit pas ici d'un jugement isolé, dans la mesure où plusieurs autres films noirs français, comme Pépé le Moko, Le Puritain ou Quai des brumes, sont régulièrement comparés, de façon dépréciative, à des films américains jugés similaires comme Scarface, The Informer (John Ford, 1935) ou encore Dead End (William Wyler, 1938). Ce procédé est manifeste chez Rebatet, qui n'a de cesse, dans ses critiques des films de Carné, de mentionner la "terrible beauté » de «ce film pourtant beaucoup plus noir encore que tous ceux de M. Carné : Le Mouchard, de l'irlandais John Ford» (Vinneuil, 17 mai 1939), pour mieux affirmer son dégoût par rapport à des films qu'il juge incapables d'élever le fait divers au rang de la tragédie : « Un artiste a le droit de traiter tous les thèmes, si noirs, si abjects soient-ils. Le Mouchard, de M. John Ford, était autrement sombre et même répugnant que Le Quai des brumes ${ }^{30}$. Mais M. John Ford dominait son sujet de haut, l'empoignait durement sans concessions » (Vinneuil, 20 mai 1938). On peut voir dans cette stratégie un signe du fait qu'en 1938, l'adjectif «noir » était déjà prêt à être réorienté dans une intention à la fois moins polémique, plus élogieuse et plus « auteuriste » à propos du cinéma américain.

\section{Conclusion}

L'étiquette générique « film noir » renvoie dès son apparition à la veille de la Seconde Guerre mondiale à un ensemble d'éléments qui se rapprochent sensiblement des traits caractéristiques que l'on associe aujourd'hui au film noir américain : un style visuel sombre proche par certains aspects du style des drames allemands des années 1920 et 1930 ; un accent mis sur le crime et le motif de la mort ; un registre mélodramatique et 
une syntaxe tragique; ou encore une différence narrative avec le modèle du récit à énigme.

Ce que Borde et Chaumeton s'attachent à définir en 1955 comme « un nouveau type de film américain » (Borde \& Chaumeton, 2004, 11) ressemble en réalité beaucoup aux films noirs français d'avant-guerre, malgré ce qu'en disent les deux critiques, et même s'il existe évidemment aussi des différences entre les deux expressions nationales du genre, qu'il n'appartient pas à cet article d'identifier ${ }^{31}$. Signe fort de cette proximité, le terme "film noir" possède des implications extrêmement similaires dans les deux contextes - la dimension polémique liée au contexte d'avant-guerre mise à part. Plus encore, on ne peut qu'être frappé, à la lecture des premiers comptes rendus mobilisant l'appellation au sujet de certains films américains, par la persistance dans les discours de nombreuses remarques, expressions ou formules couramment employées à la fin des années 1930 à propos du cinéma français: "couleur berlinoise", "atmosphère spéciale ", "sombre histoire ", "mort violente », «aventure criminelle ", «femme fatale », «fatalité du mal », etc. Enfin, même si les critiques de l'après-guerre vont peu à peu exprimer l'idée que le film noir est une forme spécifiquement américaine, il apparaît dans les deux cas que l'expression est au départ mobilisée par les critiques pour désigner une forme dont la double particularité et de dépasser les frontières nationales et de reposer en particulier sur de multiples interactions entre Paris, Berlin et Hollywood.

L'étude comparée des discours tenus à la fin des années 1930 sur le film noir français et des discours tenus à partir de 1946 sur le film noir américain montre donc que le clivage France/Amérique entériné par l'ouvrage de Borde et Chaumeton s'apparente davantage à une construction idéologiquement déterminée qu'à une réalité objective : si le Panorama du film noir américain (1941-1953), que des générations de critiques, de cinéphiles et de chercheurs ont considéré comme l'ouvrage de référence sur le sujet, a institué le film noir comme genre purement américain, il ressort clairement, au terme de l'enquête pragmatique menée dans cet article sur les usages de la dénomination «film noir» avant et après-guerre, que ce terme générique, l'un des plus communément et intimement associés avec l'imaginaire américain, a en fait servi à l'origine à la fois à désigner un ensemble de films français et une forme générique transnationale.

\section{BIBLIOGRAPHIE}

\section{Ouvrages et articles}

ALBERA, François, « 1945 : trois “intrigues” de Georges Sadoul », Cinémas, vol. 20, n² 2-3, printemps 2011, 49-85.

ANDREW, Dudley, Mists of Regret. Culture and Sensibility in Classic French Film, Princeton, Princeton University Press, 1995. 
BANTCHEVA, Denitza, Jean-Pierre Melville. De l'œuvre à l'homme (1996), Paris, Revif, 2007.

BERTHÉ-GAFFERIO, Catherine, « Le cinéma policier français de 1930 à 1950. L'âge d'or du film à énigme, le Whodunit ", dans Raphaëlle Moine, dir., Le Cinéma français face aux genres, Paris, AFRHC, 2007, 203-12.

BIESEN, Sheri Chinen, Blackout. World War II and The Origins of Film Noir, Baltimore, The Johns Hopkins University Press, 2005.

BORDE, Raymond et CHAUMETON, Étienne, Panorama du film noir américain (1941-1953) (1955), Paris, Flammarion, 2004.

BROWN, Tom, «Les remakes des films français : l'âge classique », traduit de l'anglais par Christian Viviani, dans Christian Viviani, dir., Hollywood. Les connexions françaises, Paris, Nouveau monde, 2007, 345-75.

CAROLL, David, French Literary Fascism. Nationalism, Anti-Semitism, and the Ideology of Culture, Princeton, Princeton University Press, 1995.

CHABROL, Marguerite, "Casque d'or : le sens du tragique », dans Marguerite Chabrol et Alain Kleinberger, dir., Casque d'or. Lectures croisées, Paris, L'Harmattan, 2010.

DUHAMEL, Marcel, Texte de présentation de la « Série Noire », dans Peter Cheyney, Cet homme est dangereux, Paris, Gallimard, 1945, 7.

ESCOLA, Marc, Le Tragique, Paris, Flammarion, 2002.

FAULKNER, Christopher, « Theory and Practice of Film Reviewing in France in the 1930s: Eyes Right (Lucien Rebatet and Action française 1936-1939) ", French Cultural Studies, vol. 3, n 8, juin 1992, 133-55.

FAY, Jennifer et NIELAND, Justus, Film Noir. Hard-Boiled Modernity and the Cultures of Globalisation, Londres et New York, Routledge, 2010.

HAYWARD, Susan, French National Cinema, Londres et New York, Routledge, 1993.

KRUTNIK, Frank, In a Lonely Street. Film Noir, Genre, Masculinity (1991), Londres et New York, Routledge, 2004.

MOINE, Raphaëlle, « L'évitement des genres : un fait critique ? », dans Jean-Pierre Bertin-Maghit et Geneviève Sellier, dir., La Fiction éclatée : petits et grands écrans français et francophones, vol. 1, « Études socioculturelles », Paris, INHA/L'Harmattan, 2007, 105-19.

NAREMORE, James, More Than Night. Film Noir in its Contexts, Berkeley, Los Angeles et Londres, University of California Press, 1998.

O'BRIEN, Charles, « Film Noir in France: Before the Liberation », Iris n 21, « European Precursors of Film Noir/Précurseurs européens du film noir », printemps 1996, 7-30.

ORY, Pascal, L'Aventure culturelle française, 1945-1989, Paris, Flammarion, 1989.

PASTOUREAU, Michel, Noir. Histoire d'une couleur, Paris, Seuil, 2008.

PHILLIPS, Alastair, City of Darkness, City of Light. Émigré Filmmakers in Paris 1929-1939, Amsterdam, Amsterdam University Press, 2004.

SCHRADER, Paul, « Notes on Film Noir » (1972), dans Alain Silver et James Ursini, dir., Film Noir Reader (1996), New York, Limelight Editions, 2006, 53-63.

SILVER, Alain \& WARD, Elizabeth, dir., Film Noir. An Encyclopedic Reference to the American Style (1979), New York, The Overlook Press, 1992. 
SILVER, Alain, WARD, Elizabeth, URSINI, James et PORFIRIO, Robert, dir., Film Noir. The Encylopedia, New York, The Overlook Press, 2010.

TADIÉ, Benoît, Le Polar américain. La modernité et le mal (2006), Paris, Presses Universitaires de France, 2007.

VINCENDEAU, Ginette, « Melodramatic Realism: on Some French Women's Films in the 1930 », Screen, vol. 30, n³, été 1989, 51-65.

---, « Noir is Also a French Word: The French Antecedents of Film Noir », dans Ian Cameron, dir., The Movie Book of Film Noir, Londres, Studio Vista, 1992, 49-58.

---, The Encyclopedia of European Cinema, Londres, BFI, 1995.

---, « The Art of Spectacle: The Aesthetics of Classical French Cinema », dans Michael Temple et Michael Witt, dir., The French Cinema Book, Londres, BFI, 2004, 137-152.

\section{Comptes-rendus de presse}

ANONYME, «À Quai des brumes le grand prix national du Cinéma français », Le Petit Journal, 8 juillet 1939.

ARNOUX, Alexandre, «Le Dernier tournant - Le jour se lève », Les Nouvelles littéraires, 17 juin 1939.

ASTRUC, Alexandre, « Assurance sur la mort. Le crime vient à la fin », Spectateur, 13 août 1946.

BOREL, Jacques, « Hollywood n'a pas standardisé Fritz Lang », L'Écran français, nº 49, juin 1946, $12-13$.

BOUDOT-LAMOTTE, Emmanuel, « Le Puritain », Fait, 7 décembre 1940.

BOURGEOIS, Jacques, « La tragédie policière ", La Revue du cinéma, $2^{\mathrm{e}}$ série, $\mathrm{n}^{\circ}$ 2, novembre 1946 , 70-72.

CHAMPEAUX, Georges, « Le Dernier Tournant », Gringoire, 25 mai 1939.

CHARTIER, Jean-Pierre, "Les Américains aussi font des films "noirs" ", La Revue du cinéma, $2^{\mathrm{e}}$ série, $\mathrm{n}^{\circ} 2$, novembre $1946,67-70$.

COQUET, James de, « Le jour se lève », Figaro, 14 juin 1939.

FAYARD, Jean, « Le jour se lève », Candide, 28 juin 1939.

---, « Le Facteur sonne toujours deux fois », Opéra, 19 avril 1947.

FRANK, Nino, « Un nouveau genre "policier" : l'aventure criminelle », L'Écran français, n 61 , août $1946,8-9$ et 14 .

F.N., « Le jour se lève est le film qui m'a coûté le plus d'efforts », Pour vous, n 552, 14 juin 1939, 5. GOREL, Michel, « Des gratte-ciel d'Amérique aux faubourgs parisiens », Cinémonde, $\mathrm{n}^{\circ}$ 277, 8 février 1934, 114.

HATTIER, J.J., « Le noir et le blanc », Parallèle 50, 11 ocobre 1947.

JAMESON, Amable, « Le style germanique à Hollywood », La Revue du cinéma, $2^{\mathrm{e}}$ série, $\mathrm{n}^{\circ} 6$, printemps 1947, 64-66.

MANSART, Jérôme, « Assurance sur la mort », Époque, 16 août 1946.

MONTGON, A. de, « La Bête humaine », Nom du périodique illisible, 28 janvier 1939.

ROHMER, Éric, « Redécouvrir l'Amérique », Cahiers du cinéma, n 54, décembre 1955, 11-16. 
THÉVENOT, Jean, « Quai des orfêvres », Jeunesse ouvrière, 22 novembre 1947.

VEBER, Serge, « La Bête humaine », Pour vous, n 528, 28 décembre 1938.

VINNEUIL, François, « Jenny », L'Action française, 16 octobre 1936.

---, « Pépéle Moko », L'Action française, 5 février 1937.

---, « Le Puritain », L'Action française, 21 janvier 1938.

---, « Quai des Brumes », L'Action française, 20 mai 1938.

---, « Réalisme : “Hôtel du nord” ", L’Action française, 30 décembre 1938.

---, « La Bête humaine », L’Action française, 6 janvier 1939.

---, « Le Dernier Tournant », L’Action française, 19 juin 1939.

---, « Le jour se lève », Le Petit Parisien, 1941 (date exacte illisible).

VUILLERMOZ, Émile, « La Bête humaine. Hôtel du Nord », Temps, 14 janvier 1939.

---, « Le jour se lève », Temps, 17 mai 1939.

W. L., « Le Puritain », Pour vous, n 479, 19 janvier 1938.

\section{NOTES}

1. Ma traduction, comme toutes celles de cet article, sauf indication contraire dans la bibliographie.

2. Silver reprend mot pour mot ici un paragraphe issu de l'introduction de la première version de l'encyclopédie parue en 1979. L'auteur explique lui-même cette similitude quelques lignes plus loin en soulignant que «pour l'essentiel, ce que mes co-éditeurs et moi-même pensions du film noir en 1979 n'a pas changé. En conséquence, et à l'exception de quelques brefs rajouts, la suite de cette introduction n'a pas été fondamentalement revue " (Silver, Ward, Ursini et Porfirio, $2010,15)$. Il ajoute ensuite, en reprenant à nouveau à l'identique les termes de l'introduction de 1979, que « les critiques français furent les premiers à discerner les particularités d'un certain nombre de productions américaines sorties pour la première fois en France après la Seconde Guerre mondiale » (Ibid.), sans mentionner l'émergence du terme «film noir » dans la critique française des années 1930 et son emploi initial à propos du cinéma français.

3. Ce film méconnu constitue la première adaptation cinématographique du roman noir de l'écrivain américain James M. Cain, The Postman Always Rings Twice (paru aux États-Unis en 1934, et dont la traduction française a été publiée en 1936 aux éditions Gallimard), sept ans avant la version hollywoodienne de Tay Garnett. Le roman de Cain a également donné lieu à une version italienne : Ossessione (Luchino Visconti, 1943).

4. On définit le plus souvent le « réalisme poétique » comme un « courant stylistique français des années $1930 »$ (Vincendeau, 1995, 336), bien que les traits caractéristiques les plus souvent utilisés pour en préciser les spécificités ne soient pas uniquement d'ordre esthétique et qu'ils semblent plutôt désigner un genre à part entière : dans son sens le plus courant, le terme désigne en effet « des drames urbains nostalgiques et pessimistes, généralement situés à Paris [...] Leurs récits d'amour voués à l'échec se focalisent sur des héros prolétaires au destin tragique, que Jean Gabin a le mieux incarné » (Vincendeau, 2007, 147). L'expression a toutefois pu être investie d'un sens différent selon les époques et selon les usagers qui l'ont employée: pour une analyse critique des commentaires de type historiographique tenus sur le "réalisme poétique ", voir Albera, 2011. 
5. Nous ne sommes toutefois pas en mesure de confirmer cette affirmation, étant donné que nous n'avons pas pu retrouver la trace de l'article dans lequel Paulhan aurait employé l'expression « réalisme poétique " pour la première fois (dont la référence n'est indiquée ni par Andrew ni par Phillips). Il convient donc de considérer l'origine littéraire de ce vocable avec précaution.

6. Si comme le note Tom Brown, le cinéma français est « depuis longtemps la source étrangère majeure des remakes hollywoodiens" (Brown, 2007, 346), on ne peut qu'être frappé par l'attirance de Hollywood pour le « réalisme poétique » et par le nombre important de films noirs hollywoodiens s'inspirant de films français réalisés dans les années 1930 : La Chienne (Jean Renoir, 1931), Pépé le Moko, La Bête humaine, Le jour se lève et Le Dernier tournant sont respectivement à l'origine de Scarlet Street (Fritz Lang, 1945), Algiers (John Cromwell, 1938) et Casbah (John Berry, 1948), Human Desire (Fritz Lang, 1954), The Long Night (Anatole Litvak, 1947) et The Postman Always Rings Twice.

7. Lucien Rebatet est une figure importante du paysage cinématographique, et plus largement du paysage culturel de l'époque. Antisémite convaincu au discours extrêment violent d'un côté et homme de lettres aux activités multiples (historien de l'art, critique cinématographique et musical, essayiste, romancier) de l'autre, il se présente à la fois comme l'un des principaux promoteurs d'une culture intellectuelle fasciste qui aura un impact considérable sur les mentalités de son temps (Carroll, 1995) et comme un des critiques de cinéma les plus productifs, les plus talentueux et les plus influents de l'époque : « Il était l'un des plus anciens critiques de films professionnels de la décennie, possédant une connaissance précise du cinéma, de ses institutions, de ses pratiques et de son histoire, ainsi que des films, des cinéastes, des genres et des styles. Entre 1936 et 1939, Rebatet écrivit approximativement quarante critiques par an pour L'Action française, dont chacune pouvait évoquer de un à trois films » (Faulkner, 1992, 143-144).

8. Quai des orfêvres a par exemple pu être décrit comme un film d'un «réalisme absolu ne redoutant pas le noir" (Hattier, 11 octobre 1947), ou comme un «film noir qui se rattache aux Carné d'avant-guerre, Quai des brumes, Le jour se lève » (Thévenot, 22 novembre 1947).

9. Dans leur encyclopédie du film noir, Alain Silver et Elizabeth Ward expliquent que «la définition du film noir utilisé dans ce travail [...] est assez similaire à celle proposée par l'ouvrage séminal sur le sujet, à savoir le Panorama du film noir américain de Raymond Borde et Étienne Chaumeton, initialement publié en 1955 ». Ils ajoutent ensuite que cet ouvrage constitue une " référence pour tout travail ultérieur cherchant à définir le même sujet » (Silver et Ward, 1992, 372).

10. On pourrait d'ailleurs contester l'argument selon lequel «l'onirisme et l'insolite font complètement défaut dans ces films des années 1936-1938 » : comme le nom que l'historiographie du cinéma lui a donné l'indique, le «réalisme poétique " mêle l'authenticité à l'abstraction lyrique, ce qui découle en partie de l'hybridité culturelle de ces films français souvent éclairés par des chefs-opérateurs allemands ; un film comme Quai des brumes (dont la lumière a été créée par Eugen Shüfftan) construit un style oscillant constamment entre réalisme « documentaire » et effet esthétisant.

11. Pour effectuer cette étude pragmatique, nous nous sommes principalement appuyé sur les comptes-rendus critiques conservés à la Bibliothèque Nationale de France. La collection « Rondel », en particulier, qui se présente sous la forme de recueils d'articles de presse consacrés à des films précis, nous a permis de consulter certaines références qui auraient autrement été introuvables sur un petit nombre de films noirs français des années 1930. Nous souhaitons toutefois attirer l'attention du lecteur sur la nature fatalement incomplète d'un tel travail, portant sur des sources aussi anciennes. En effet, seul un très petit nombre de critiques ont effectivement été conservées, certaines sont amputées, et pour un grand nombre de films de cette période, il n'existe que très peu voire dans certain cas pas du tout de ressources datant de 
l'époque disponible pour le chercheur. On ne peut donc présenter ici que les résultats, les plus rigoureux possibles, d'une étude fatalement incomplète.

12. Sous l'influence de commentateurs tels que Paul Schrader, Janey Place et Lowell Peterson, le style visuel est progressivement apparu comme un des critères les mieux à même d'unifier les différents types de films américains susceptibles d'être qualifiés de «films noirs ». Ainsi, en 1972, dans ses célèbres « Notes on Film Noir », Paul Schrader n'hésite pas à affirmer que «le film noir était avant tout un style » (Schrader, 2006, 63). Les analyses les plus récentes invitent à relativiser cette affirmation et à prendre conscience de la diversité des marqueurs esthétiques composant ce que l'on a coutume d'appeler le «style noir » (voir par exemple Fay et Nieland, 2010). Il n'en demeure pas moins que le style constitue incontestablement un élément fortement identifiant du genre (à ce sujet, voir Biesen, 2005).

13. Pastoureau, 2008

14. "Je ne sais s'il est prudent de cultiver avec autant de soin ce sombre lyrisme » (Vuillermoz, 14 janvier 1939).

15. «Et c'est une série d'images magnifiques, traitées avec une intelligence, un goût et cette sombre poésie dont Marcel Carné possède le secret » (Vuillermoz, 17 mai 1939).

16. Les deux cinéastes ont chacun réalisé durant leur séjour en France dans les années 1930 au moins un drame criminel qu'il semble tout à fait pertinent de redéfinir aujourd'hui a posteriori comme un film noir français : Liliom (1934) dans le cas de Lang; Tumultes (réalisé en 1932, et qui constitue la version française du film allemand tourné la même année par le même réalisateur, Stürme der Leidenschaft) et Pièges (1939) dans le cas de Siodmak.

17. Jameson écrit à propos de The Killers que l'histoire n'aurait eu aucun intérêt particulier «si elle n'avait pas une couleur spéciale; avec des tons empruntés tantôt à Lang, tantôt à Orson Welles, mais aussi avec une sensibilité particulière quant à la façon de présenter les faits au spectateur » (Jameson, 1947, 65, je souligne). L'expression rappelle nombre de comptes-rendus publiés dans les années 1930 à propos des films noirs français, comme par exemple l'article précédemment cité du Petit Journal à propos de Quai des brumes, qui assimilait le film noir à un « genre très spécial »(Anonyme, 8 juillet 1939).

18. "L'étude des bas-fonds apporte à nos cinéastes et à nos dramaturges des éléments de pittoresque facile qui expliquent leur engouement pour cette formule industrialisée» (Vuillermoz, 14 janvier 1939).

19. Les italiques sont de l'auteur, et révèlent peut-être, comme O'Brien le suggère $(1996,11)$, la nouveauté du terme dans ce texte datant du mois de janvier 1938. On notera au passage qu'il s'agit là à notre connaissance de l'unique critique positive de Rebatet sur un film noir français.

20. On peut noter ici l'emploi de l'expression "mort violente ", qui sera reprise après-guerre dans une formule célèbre par Nino Frank pour définir la singularité du film noir américain : « il y aurait d'amusantes conclusions à tirer de ce remplacement du dynamisme des poursuites et de l'idylle mouvementée, par le dynamisme de la mort violente» (Frank, 1946, 9). Borde et Chaumeton reprendront cette formule dans le chapitre liminaire de leur Panorama du film noir américain consacré à la définition du genre: "Dynamisme de la mort violente", disait Nino Frank, et l'expression paraît excellente » (Borde et Chaumeton, 2004, 15-16).

21. Dans un texte écrit pour accompagner le premier roman paru dans la collection "Série Noire » des éditions Gallimard en septembre 1945-Cet homme est dangereux de Peter Cheyney-, Marcel Duhamel définit le roman noir anglo-saxon comme un " genre nouveau » en rupture avec la fiction policière à l'anglaise : «Le roman détective a subi depuis quelques années dans les pays anglo-saxons une évolution suffisamment marquée pour que l'on puisse maintenant parler d'un genre nouveau. L'intérêt cesse de se porter sur la solution de l'énigme » (Duhamel, 1945, 7). Cette argumentation va être reprise et développée par une grande partie des nouveaux usagers du terme « film noir », à commencer par Nino Frank, dont le celèbre article fondateur s'intitule « Un nouveau genre "policier" : l'aventure criminelle». 
22. "Cette nouvelle orientation du film policier appelle un commentaire. Tout d'abord, elle n'est pas une nouveauté comme on l'a dit ici ou là. Un film de M. Chenal, "Alibi”, procédait du même scénario » (Mansart, 16 août 1946). L'auteur évoque ici le film L'Alibi (Pierre Chenal, 1937).

23. Nous empruntons la notion de « mécanisme tragique » à Escola, 2002.

24. Voir Vincendeau, 1989 ; Hayward, 1993.

25. Les critiques d'après-guerre évoquent majoritairement la noirceur des films américains dans des termes élogieux en y voyant une qualité artistique. Chez certains, la dimension tragique des films est rattachée à une conception existentialiste de l'existence, c'est-à-dire à une façon de regarder avec lucidité le monde contemporain : dans un texte célèbre datant de 1955, dans lequel le jeune critique des Cahiers du cinéma soutient que « c'est au genre policier que le film américain doit sa meilleure inspiration", Rohmer écrit que "les scénaristes d'Hollywood ont su nous dessiner l'image d'un monde où, si le Bien ou le Mal existent, les frontières [...] ne sont que des sinuosités ou enclaves inattendues » (Rohmer, 1955, 14). Pour d'autres, c'est le cynisme et l'aspect savoureusement corrosif du «noir» qui constitue un des principaux attraits des films américains : pour Alexandre Astruc, « un nouvel art du roman policier, fait de violences glacées et d'indifférence cruelle, remonte sur toutes les façades de cinéma. [...] Le tout raconté avec un cynisme joyeux, assez proche de l'humour noir cher aux surréalismes, qui fait des richochets dans les mers de sang et joue aux osselets avec des crânes humains » (Astruc, 1946). Dans les deux cas, l'éloge de la noirceur marque un contraste abyssal avec la rhétorique polémique d'avantguerre.

26. Certains films sont également vus comme les successeurs du «naturalisme » de Zola, ce qui chez les critiques d'extrême droite constitue une infamie absolue: dans sa critique de La Bête humaine, Rebatet ne manque pas de rappeler que « s'il est un cas sur lequel toute L'Action française tombe d'accord, c'est certainement celui de Zola », avant de qualifier l'esthétique naturaliste du film d'« infirmité » et de stigmatiser "l'étude de l'abjection humaine étalée à chaque instant devant nos yeux » (Vinneuil, 6 janvier 1939).

27. Selon The Encyclopedia of European Cinema, le Strassenfilme peut se définir comme un "genre

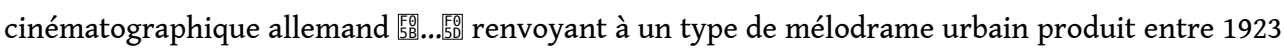
et 1930, dans lequel un protagoniste (généralement masculin) de la classe moyenne s'égare dans "la rue" en cherchant à échapper à l'ennui et au confinement moral de l'existence bourgeoise, tandis qu'un protagoniste (généralement féminin) de la classe laborieuse tente d'échapper au milieu des bas-fonds ». Le Kammerspielfilm est " un genre allemand désignant des films produits au début des années 1920 qui puisent dans les conventions du théâtre allemand contemporain. Typiquement, l'intrigue est un drame réaliste représentant des domestiques ou des membres des classes laborieuses qui connaissent un destin fatal à travers le meurtre ou le suicide » (Vincendeau, 1995, 235 et 409).

28. Le titre original est Die freudlose Gasse (Georg Wilhelm Pabst, 1925).

29. Si les historiens attribuent souvent l'invention de la catégorie de «film noir américain » à l'article de Nino Frank paru dans L'Écran français en août 1946, c'est à notre connaissance deux mois plus tôt, dans le numéro 49 de la même revue, daté du 5 juin 1946, mais sous la plume de Jacques Borel-qui est alors le correspondant à Londres de L'Écran français-que l'on trouve sa première occurrence pour exprimer l'idée d'un renouveau dans la production cinématographique américaine.

30. Rebatet emploie ici le titre original du film de Carné, Le Quai des brumes (qui est également le titre du roman de Mac Orlan dont le film est l'adaptation), que l'on appelle aujourd'hui systématiquement Quai des brumes (terminologie que nous avons donc adoptée dans cet article).

31. En relativisant l'affirmation de Borde et Chaumeton, selon laquelle le film noir américain constitue un "nouveau type de film américain », nous n'entendons pas nier les singularités propres aux films américains ni les innovations que le cinéma hollywoodien a apporté au genre : il s'agit simplement pour nous de contester un clivage arbitraire, et de mettre en lumière des 
liens manifestes entre la France et Hollywood, qui ont été occultés par l'historiographie traditionnelle du film noir.

\section{RÉSUMÉS}

Selon l'historiographie du genre, l'appellation «film noir » a été forgée en France en 1946 pour caractériser des produits filmiques américains. Depuis cette date, il est de coutume de réserver ce terme générique aux seuls films hollywoodiens et de considérer ce genre comme une spécificité américaine. Dans les années 1990, des chercheurs anglo-saxons ont certes entrepris de dissocier le genre du seul cinéma américain : Charles O'Brien a ainsi montré que l'étiquette « film noir » a initialement été employée en France avant la Seconde Guerre mondiale, pour parler d'un ensemble de films français proche $d u$ "réalisme poétique ", avant d'être "réorientée " aprèsguerre pour désigner la fiction américaine. Mais ces travaux demeurent méconnus en France, où le film noir continue d'être perçu comme américain. Face à la persistance de cette vision trompeuse et à l'oubli de la tradition «noire » française, il est nécessaire de mettre à jour les implications de la dénomination dans la France des années 1930. Que signifiait l'expression « film noir» pour la critique de l'époque? Dans quelle mesure était-elle utilisée pour désigner une forme de fiction criminelle transnationale? L'histoire oubliée de la genèse française de cette célèbre appellation critique peut-elle nous renseigner sur l'identité et la dimension internationale du film noir?

According to the established historiography, the generic label "film noir" was used in France in 1946 to refer to a series of Hollywood crime fictions produced in the 1940s and 1950s. Since then, the term has been exclusively associated with Hollywood pictures and "film noir" has been considered a specifically American form. In the 1990s, North American and British scholars started to re-evaluate film noir and show that this genre was not exclusive to American cinema: in fact, Charles O'Brien revealed that the label "film noir" had first been used in France before the war to describe a group of French films that are more or less the same ones we now identify as "poetic realism"; he then went on to refer to a new tendency in post-World-War-II Hollywood cinema. However, his work has, for the most part, gone unrecognized in France, where film noir is still seen as purely American. In the face of the persistence of this distorded vision and the progressive sinking into oblivion of the French "noir" tradition, it is necessary to replace the term in its original context, that of 1930s France. What did the term "film noir" mean for film reviewers of the time? To what extent was it used to describe a certain kind of transnational criminal fiction? Can the forgotten history of this famous term's French origins enlighten us on film noir's identity and international dimension?

\section{INDEX}

Keywords : 1930s, Film criticism, Film noir, French cinema, Genre, Transnational cinema

Mots-clés : Années 1930, Cinéma français, Critique cinématographique, Film noir, Genre, Transnational 
AUTEUR

THOMAS PILLARD

Université Michel de Montaigne Bordeaux 3 